\title{
Some aspects of entropic gravity in the presence of a noncommutative Schwarzschild-deSitter black hole
}

\author{
S. Hamid Mehdipour* \\ Department of Physics, Lahijan Branch, Islamic Azad University, P. O. Box 1616, Lahijan, \\ Iran
}

\begin{abstract}
We study some features of entropic force approach in the presence of a noncommutative Schwarzschild-deSitter black hole. In this setup, there exists a similarity between the small and large scales. There are two finite cut-off in very short and long distances wherein the force and energy graph stop abruptly at those scales. We find that the existence of a deSitter core around the origin, induced by noncommutativity, in addition to a standard deSitter background at large scale may lead to a violation of the equivalence principle. Finally in order to directly observe the finite cut-off at short-scale gravity, caused by noncommutativity quantum fluctuations, we derive an effective gravitational constant.
\end{abstract}

PACS: 04.70.Dy, 04.50.Kd, 05.70.-a, 02.40.Gh, 04.20.Dw

Key Words: Black Hole Thermodynamics, Noncommutative Geometry, Holographic Screens, Entropic Force, Cosmological Constant, Equivalence Principle

*mehdipour@liau.ac.ir 


\section{Introduction}

There are many proofs signifying a profound relation between thermodynamics and the general theory of relativity. Discovery of black hole radiation demonstrated that the black hole behaves as a thermal system [1]. The thermodynamic laws of black holes suggest a meaningful connection between gravity and thermodynamics [2]. In 1995, Jacobson derived the Einstein field equation from the first law of thermodynamics [3]. Recently, Padmanabhan attained the Einstein field equation by uniting the equipartition law of energy and the holographic principle [4]. In addition Verlinde illustrated gravity as an entropic force, as a result of alterations in the information related to the locations of material bodies [5]. He acquired an effective force acting on a test mass coming near to a holographic screen, caused by the alteration of entropy on the screen, which satisfies the Newton's second law for gravitational force. Verlinde's proposal has extensively been debated in the literature [6].

Recently, we investigated some aspects of the entropic essence of gravity in the presence of noncommutative Schwarzschild [7] and Reissner-Nordström [8] black holes by performing the method of coordinate coherent states representing smeared structures. This method of noncommutativity is the so-called noncommutative geometry inspired model (for a review see [9]). The eliciting of metrics for noncommutative geometry inspired black holes is established upon the feasible running of the minimal observable length in general relativity. Based on this new model of noncommutativity of coordinates, which performs the Gaussian distribution of coherent states, the Einstein tensor in gravity field equations remains intact but the energy-momentum tensor takes a new form. In fact, due to the emergence of extreme energies at short distances of a noncommutative manifold, the effects of manifold quantum fluctuations become visible and prohibit any measurements to find a particle position with an accuracy more than an inherent length scale, such as the Planck length, and this means that the concept of locality is violated [10]. Accordingly, a point-like particle in a noncommutative spacetime is no longer modelled by a Dirac-delta function distribution, but will be characterized as a smeared-like particle by a Gaussian distribution of minimal width $\sqrt{\theta} \dagger$, where $\theta$ is the smallest fundamental unit of an observable area in the noncommutative coordinates, beyond which coordinate resolution is ambiguous.

\footnotetext{
${ }^{\dagger}$ The value of $\sqrt{\theta}$ without appearing the extra-dimensions scenarios is a value of the order of the Planck length, i.e. $\sqrt{\theta} \sim 10^{-33} \mathrm{~cm}$.
} 
In addition, noncommutative solutions for black holes smoothly incorporate the deSitter core around their origin into an ordinary metric of the black hole far away from its origin [11]. Thus, as an impressive outcome of this noncommutative solution, the curvature singularity at the origin of black holes is eliminated. In lieu of the curvature singularity, a regular deSitter vacuum state will be formed regarding the influence of the strong quantum fluctuations at short distances in a noncommutative manifold. This approach descends to a usual metric at large distances where the demeanor of the minimal length is insignificant, while providing new physics which appears at short distances.

On the other hand, since in the noncommutative geometry inspired solutions the existence of a deSitter core in the centre of black holes prohibits their collapse into a singular case, it can shed more light on the issue of the quantum stability of the deSitter space and accordingly, the rate of the Planck size black holes production on the inflationary background of the universe [12]. In inflationary epochs, the universe is well delineated by the deSitter geometry. The accelerating phase in the inflationary era of the universe was initiated as a plan to find a solution to the problems in the standard big-bang theory [13. The most significant observational development in cosmology is the conclusion of the cosmic accelerating expansion of the universe which was first declared in 1998, based on Supernova data and cosmic microwave background observations [14]. These data mention the appearance of some background form of the energy with a negative pressure. It is possible to illustrate this energy through a positive cosmological constant and quintessence fields. This means that, when taking objects like black holes into account one can presume the emergence of an effective, positive cosmological constant. In this paper, we would like to extend our previous work [7] to a deSitter background caused by a cosmological constant. It is clear that, in light of the facts mentioned above, the interest in considering the noncommutative Schwarzschild-deSitter black hole (NC SdS BH) becomes natural.

\section{Noncommutative Schwarzschild-deSitter Metric}

The NC SdS BH solution obtained by Mann and Nicolini [12] is given by the following metric $甘$,

$$
d s^{2}=-N(r) d t^{2}+N^{-1}(r) d r^{2}+r^{2} d \Omega^{2},
$$

\footnotetext{
${ }^{\ddagger}$ We use units with the following definitions: $\hbar=c=k_{B}=1$.
} 
where

$$
N(r)=1-\frac{2 G M_{\theta}}{r}-\frac{\Lambda}{3} r^{2}
$$

In the above, the cosmological constant is $\Lambda=3 / l^{2}$, and $l$ is the cosmological length associated with the $\Lambda$. The smeared mass distribution $M_{\theta}$ is found to lead to the result

$$
M_{\theta}=M\left[\mathcal{E}\left(\frac{r}{2 \sqrt{\theta}}\right)-\frac{r}{\sqrt{\pi \theta}} e^{-\frac{r^{2}}{4 \theta}}\right] .
$$

For the commutative case, $r / \sqrt{\theta} \rightarrow \infty$, the smeared-like mass descends to the point-like mass, i.e. $M_{\theta} \rightarrow M$, and one recovers the standard SdS metric. In fact, this is the regime where noncommutative fluctuations are insignificant and the background geometry may well characterized through a smooth differential manifold. However for the noncommutative case, $r \rightarrow \sqrt{\theta}$, the NC SdS metric deviates crucially from the standard one and provides novel physics at short distance regime.

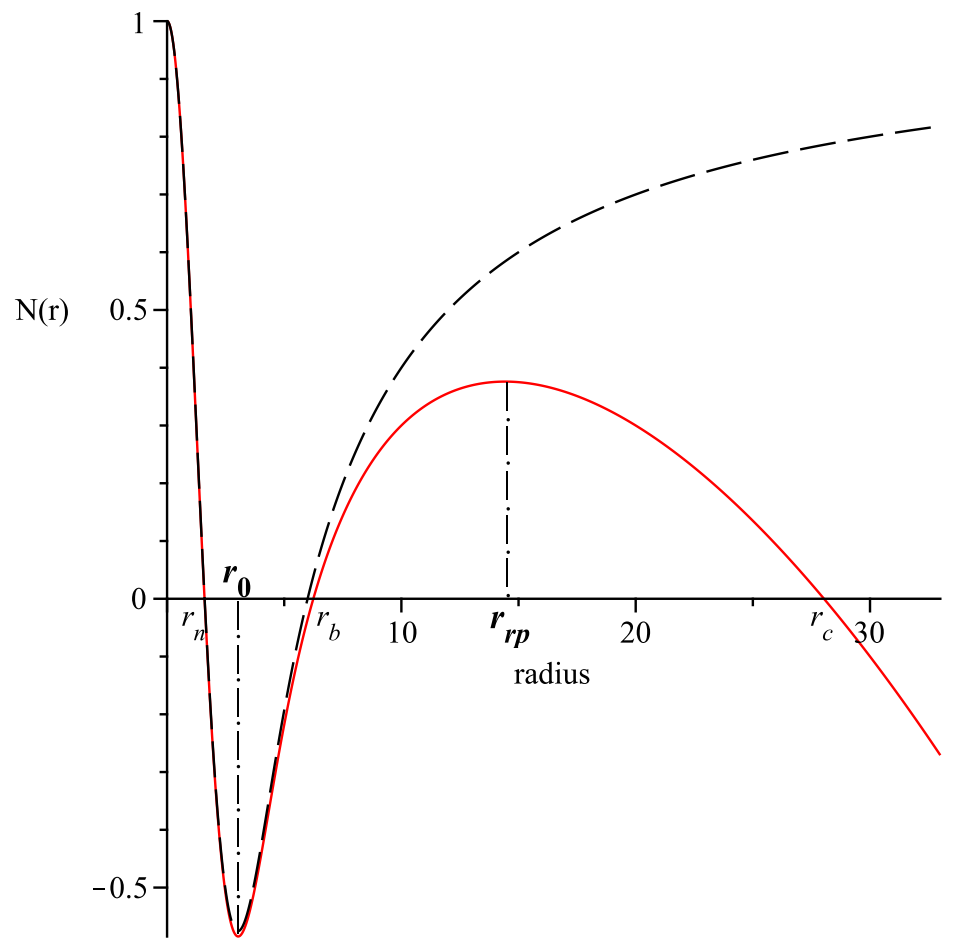

Figure 1: The function $N(r)$ versus the radius, $r / \sqrt{\theta}$ for $M=3.0 \sqrt{\theta} / G$. The solid line corresponds to the $\mathrm{NC} \mathrm{SdS} \mathrm{BH}$ for $\Lambda / 3=10^{-3} / \theta$, and the dashed line corresponds to the NC Schwarzschild $\mathrm{BH}(\Lambda=0)$.

For further details, we draw the temporal component of the metric (1), $N(r)$, as a function of $r / \sqrt{\theta}$ (see Fig. 1), for $M=3.0 \sqrt{\theta} / G$, in two cases: a) deSitter background for 
$\Lambda / 3=10^{-3} / \theta$ and; b) asymptotically flat space. The solid line presented in Fig. 1 shows the possibility of having three distinct horizons at a typical case when the mass of the black hole is larger than the minimal nonzero mass $M_{0}$ but smaller than the Nariai mass $M_{N}$. Note that, for $M>M_{N}$, there is no timelike Killing vector, and for $M<M_{0}$, there is no black hole [12]. Intersections on the radius axis lead to radii of the event horizons. For the case a) we have three horizons, an inner (or noncommutative) $r_{n}$ and an outer black hole horizon $r_{b}$ and a cosmological event horizon $r_{c}$. But, for the case b) there are only two horizons, a noncommutative and a black hole horizon (see the dashed line). In comparison with the commutative one, for $M \gg M_{0}$, the noncommutative horizon tends to zero, while the black hole horizon reaches the Schwarzschild value, $r_{b} \rightarrow 2 M$.

According to the $\mathrm{NC}$ approach [12], due to the presence of strong quantum fluctuations in a noncommutative manifold, a regular behaviour at the origin proves natural. Since there exists a outward push caused by noncommutativity quantum fluctuations, the metric (1) is well characterized close to the origin by a deSitter geometry, this can be illustrated by a quantum pressure that is related to the cosmological constant in deSitter universe. As a result, an effective cosmological constant corresponding to the deSitter type solution, i.e. $N(r) \approx 1-\Lambda_{\mathrm{eff}} r^{2} / 3$, can be generated by using the asymptotic form of the metric (1) at short distances as follows:

$$
\Lambda_{\mathrm{eff}}=\Lambda+\frac{M G}{\sqrt{\pi \theta^{3}}},
$$

which is used to illustrate the accelerating expansion of the universe. As mentioned above, the regularisation is due to a local deSitter spacetime caused by the standard deSitter background, plus the noncommutative fluctuations.

The authors of Ref. [15] investigated the entropic formulation in the presence of the SdS BH as a model of multiple holographic screens. Due to the vanishing of the UnruhVerlinde temperature at the Bousso-Hawking reference point [16], they considered two regions separated by zero temperature barrier as thermodynamically isolated systems in a static geometry setup, and finally applied independently the Verlinde's entropic formalism to each region. In this work, we utilize the Bousso-Hawking reference point to observe the temperature on the holographic screens. However, we only consider the internal region restricted by surfaces at $r=r_{0}$ and $r=r_{r p}$, i.e. the pattern of the metric for $r_{0} \leq r \leq r_{r p}$, where $r_{0}$ and $r_{r p}$ are the minimal nonzero radius and the reference point radius, respectively (see Fig. 1). We will explain this issue in the next section. 


\section{Verlinde's Entropic Formalism}

In the curved spacetime, to look for a time-like Killing vector $\xi_{\alpha}$ of the NC SdS BH which is asymptotically de Sitter space, we consider a normalization constant $\sigma$ for the $\xi_{\alpha}$, where

$$
\xi_{\alpha}=\sigma\left(\partial_{0}\right)_{\alpha} .
$$

In the asymptotically flat spacetime, the standard Killing vector normalization, i.e. $\sigma=1$, is recovered. In order to prevent a complication in taking the normalization of Killing vector, we use the normalization proposed by Bousso and Hawing [16] so that the norm of the Killing vector is one at the area where the gravitational attraction and the cosmological repulsion cancel each other and thus the force vanishes. Since SdS space is not asymptotically flat, they placed a reference point in the radial direction such that it can fulfill a role of a point at infinity in an asymptotically flat spacetime. Moreover, the temperature at this point vanishes, and any thermal exchanges cannot transpire through the reference point. Therefore, a thermally insulating wall is made at that region.

Since we need to distinguish holographic screens $\Omega$ at surfaces of constant redshift and in order to clarify a foliation of space, the generalized form of the Newtonian potential $\phi$ and the acceleration $a^{\alpha}$ in the general relativistic framework can be written as

$$
\begin{gathered}
\phi=\frac{1}{2} \log \left(-g^{\alpha \beta} \xi_{\alpha} \xi_{\beta}\right), \\
a^{\alpha}=-g^{\alpha \beta} \nabla_{\beta} \phi,
\end{gathered}
$$

Using the Killing equations $\partial_{\alpha} \xi_{\beta}+\partial_{\beta} \xi_{\alpha}=2 \Gamma_{\alpha \beta}^{\gamma} \xi_{\gamma}$, here $\alpha, \beta, \gamma$ run from 0 to 3, with the condition of static spherically symmetric $\partial_{0} \xi_{\alpha}=\partial_{3} \xi_{\alpha}=0$, and also the normalization mentioned above, the gravitational potential for the $\mathrm{NC} \mathrm{SdS} \mathrm{BH}$ is found to have the form

$$
\phi=\frac{1}{2} \log \left(\sigma^{2} N(r)\right),
$$

where $e^{\phi}$ is the redshift factor and is equal to one at the the Bousso-Hawking reference point. The Unruh-Verlinde temperature on the screen can be written in the form

$$
T=-\frac{1}{2 \pi} e^{\phi} n^{\alpha} a_{\alpha}=\frac{e^{\phi}}{2 \pi} \sqrt{g^{\alpha \beta} \nabla_{\alpha} \phi \nabla_{\beta} \phi},
$$

where $n^{\alpha}$ is a unit vector which is defined as

$$
n^{\alpha}=\frac{\nabla^{\alpha} \phi}{\sqrt{g^{\alpha \beta} \nabla_{\alpha} \phi \nabla_{\beta} \phi}} .
$$


The unit vector $n^{\alpha}$ is normal to the holographic screen and to the $\xi_{\alpha}$. The existence of the expression $e^{\phi}$ in Eq. (91) is due to the fact that the temperature is measured with respect to the reference point. In our case, the reference point is placed at the region between the black hole horizon and the cosmological event horizon wherein the force is zero. The Unruh-Verlinde temperature for the NC SdS BH has the form

$$
T=\sigma \frac{N^{\prime}(r)}{4 \pi}=\frac{\sigma}{2 \pi}\left(\frac{G M_{\theta}}{r^{2}}-r h(r)\right),
$$

where the prime abbreviates $d / d r$, and

$$
h(r)=\frac{G M}{2 \sqrt{\pi \theta^{3}}} e^{-\frac{r^{2}}{4 \theta}}+\frac{\Lambda}{3} .
$$

The energy on the holographic screen $\Omega$, according to the equipartition law of energy, is immediately written as

$$
E=\frac{1}{4 \pi} \int_{\Omega} e^{\phi} \nabla \phi d A=2 \pi r^{2} T,
$$

where $A$ is the area of the screen. For the energy on the NC SdS screen, one can find

$$
E=\sigma\left(G M_{\theta}-r^{3} h(r)\right) .
$$

The entropic force is therefore given by

$$
F_{\alpha}=T \nabla_{\alpha} S,
$$

where $\nabla_{\alpha} S=-2 \pi m n_{\alpha}$, is the change in entropy for the test mass at fixed position nearby the screen. Ultimately, the entropic force in the presence of the NC SdS BH becomes

$$
F=\sqrt{g^{\alpha \beta} F_{\alpha} F_{\beta}}=\sigma\left(\frac{G M_{\theta} m}{r^{2}}-m r h(r)\right) .
$$

The numerical results of the entropic force and the energy versus the radius for two situations, a deSitter background and the asymptotically flat spacetime, are shown in Figs. 2 and 3, respectively. We notice that all of Figs. 1, 2 and 3 emphasize the region between two boundaries at radii $r_{0}$ and $r_{r p}$ such that the temperature, force and energy vanish on these boundaries. As can be seen from last two figures, the force and energy graph cut off abruptly at some finite $r$ at both small and large scales.

About the small scales, as we have already mentioned in Refs. [7, 8], due to a negative quantum pressure induced by the coordinate noncommutativity at small scales, the case of $r<r_{0}$ leads to some out of the ordinary dynamical features like negative entropic force, 


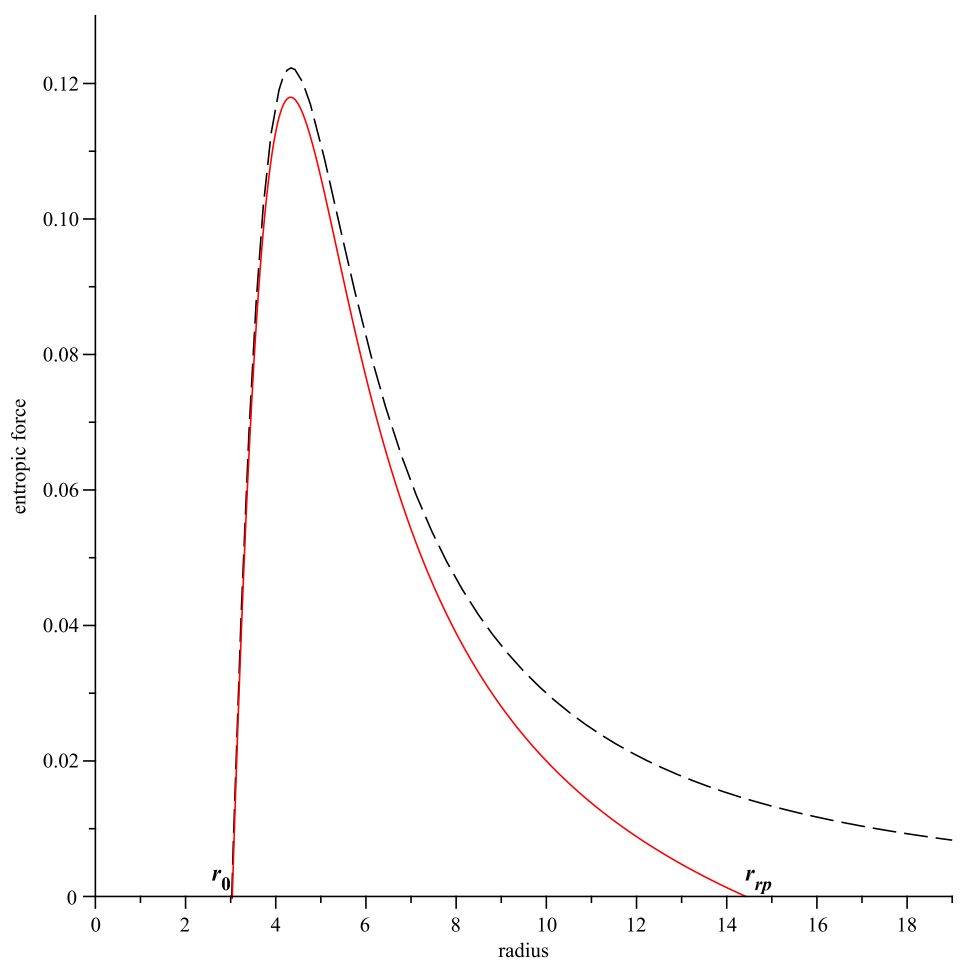

Figure 2: The entropic force $F$ versus the radius, $r / \sqrt{\theta}$. We have set $M=3.0 \sqrt{\theta} / G$. The solid line represents the entropic force in a deSitter background for $\Lambda / 3=10^{-3} / \theta$. The dashed line represents the entropic force of the NC Schwarzschild BH in asymptotically flat space.

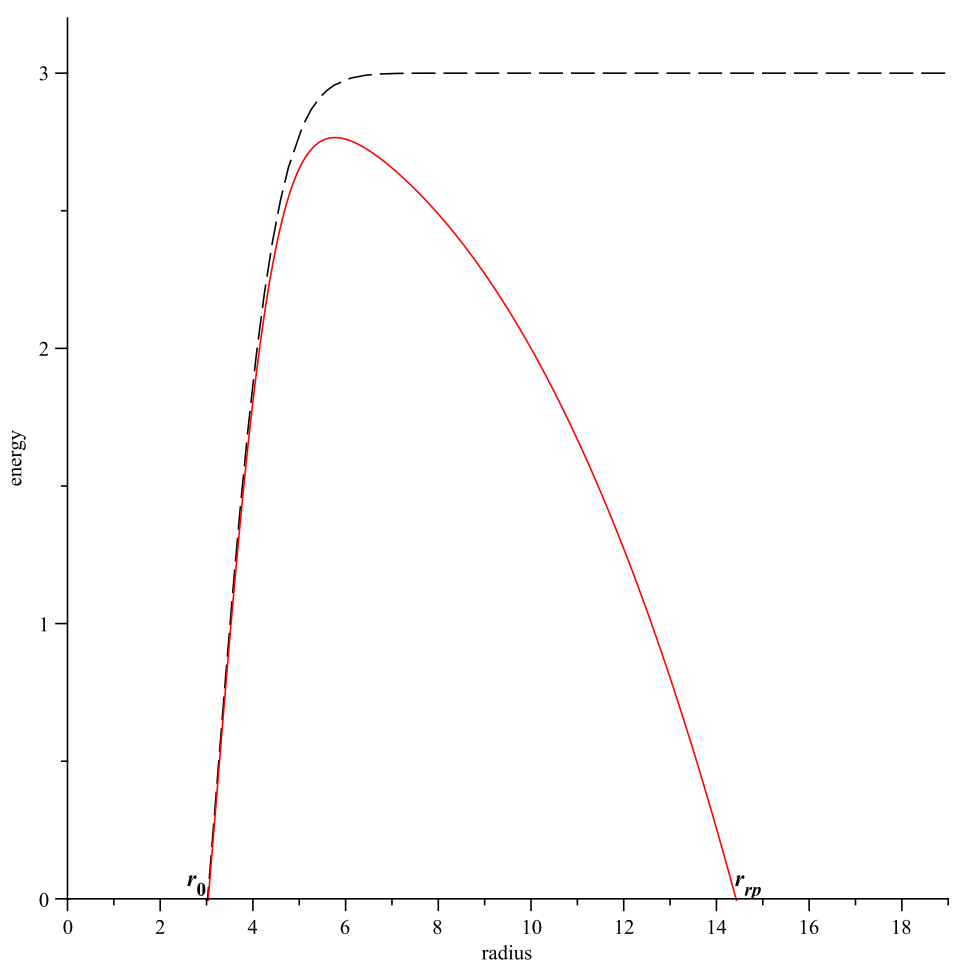

Figure 3: The energy, $E / \sqrt{\theta}$, versus the radius, $r / \sqrt{\theta}$. We have set $M=3.0 \sqrt{\theta} / G$. The solid line corresponds to the $\mathrm{NC}$ SdS BH for $\Lambda / 3=10^{-3} / \theta$, and the dashed line corresponds to the NC Schwarzschild BH $(\Lambda=0)$. 
i.e. gravitational repulsive force, and negative energy. According to the original work proposed by Nicolini et al (see the first reference of [11]), if we choose that the original mass is less than the minimal mass $M_{0}$, or the screen radius to be less than the radius of the smallest holographic surface at the Planckian regime, there cannot be a black hole and no temperature can be defined, thus the ultimate zero temperature configuration can be observed a black hole remnant at the region where the Hawking emission stops abruptly; as a result, in agreement to Verlinde's entropic formalism, the behavior of the entropic force is similar to the temperature. Moreover if $r<r_{0}$, we encounter with the unusual case of $E<0$ which is nonphysical, so the existence of finite cut-off at small scales is credible and one can make the requirement that $E \geq 0$.

There is also a same reason for the large scales. In fact, the pattern of the metric for very short distances has a similarity to the pattern of the metric for very long distances. Here we imply that the standard deSitter background at large scales may prevent a measurement to find the particle position beyond the reference point radius, a maximal observable length, in exactly the same way that the existence of a deSitter core in the centre of the black hole yields a outward push to prevent its collapse into a singular case. Therefore, it is impossible to set up a measurement to find more accurate particle position than $r_{0}$. This means that if $r$ is too small or large, as the test mass $m$ comes close to the screen, the lessening in screen entropy will produce a repulsive force. Thus, it is not necessary to consider the total system and one can ignore the patterns of the metric for $r<r_{0}$ and $r>r_{r p}$. Consequently, we apply the circumstance that the screen radius is bigger than the radius of the smallest holographic surface but is smaller than the radius of the Bousso-Hawking reference point.

As mentioned above, in agreement to our previous works for small scales [7, 8], Figs. 2 and 3 show that the entropic force and energy on the holographic screens with radii $r_{0}$ and $r_{r p}$ are zero. This is an important result due to the fact that $r_{0}$ and $r_{r p}$ are, respectively, radii of smallest and largest holographic screens, then they cannot be probed through the test mass that is located on a very short or long distance from the source. Accordingly, the conventional formulation of gravity is contravened in both small and large scales when the screen radius comes near the $r_{0}$ or $r_{r p}$. In other words, the test mass cannot recognize any gravitational field in two situations: i) when it is located at a minimal distance from the source mass; ii) when it is located at a maximal distance from the source mass. The circumstance adopted by the first situation manifestly contravenes the entity of the exclusively gravitational interaction for an inert remnant of the black hole. Black hole residues 
as crucial physical entities are profoundly confirmed in the quantum gravity literature when quantum gravitational fluctuations are exposed. As an example, when generalized uncertainty principle is taken into consideration, the total evaporation of black holes is banned and there would be massive but inert residues including the exclusively gravitational interactions [17. Our approach proves that the black hole remnants are totally inert with no gravitational interactions. This enables one to locally mark a difference between a uniform acceleration and a gravitational field. When one reaches the smallest fundamental unit of a holographic screen with radius $r_{0}$ one conflict with the equivalence principle (EP) of general relativity because there is now an essential distinction between the gravitational and inertial mass. In fact, contrary to the inertial mass, the gravitational mass in the black hole remnant possesses no gravitational field which is recognized to be zero. Therefore, there may be a violation of the EP at small scales owing to the existence of a deSitter core around the origin. In a similar manner, one can imagine that an evident violation of the EP may occur owing to the existence of a standard deSitter background at large scales. This means that it may be possible to observe a distinction between the gravitational and inertial mass in a locally frame of reference at a cosmic size. Such an argument, though perhaps too far to be perceivable directly, could in principle leaves a trace of a unified theory at all length scales.

Let us now return to Eq. (16) and consider it as

$$
F=\sigma\left(\frac{G_{\mathrm{eff}^{M m}}}{r^{2}}-\frac{m \Lambda}{3} r\right) .
$$

In the same manner, energy becomes

$$
E=\sigma\left(G_{\mathrm{eff}} M-\frac{\Lambda}{3} r^{3}\right)
$$

where $G_{\text {eff }}$ is defined as an effective gravitational constant which is written as

$$
G_{\mathrm{eff}}=G\left[\mathcal{E}\left(\frac{r}{2 \sqrt{\theta}}\right)-\frac{r}{\sqrt{\pi \theta}} e^{-\frac{r^{2}}{4 \theta}}\left(1+\frac{r^{2}}{2 \theta}\right)\right] .
$$

From the result (19) we can observe that the effective gravitational constant incorporates effects of the noncommutativity of coordinates and depends on the $r / \sqrt{\theta}$ so that in the commutative case, $r / \sqrt{\theta} \rightarrow \infty$, we have the usual gravitational constant, i.e. $G_{\text {eff }} \rightarrow G$. Thus, we see that the noncommutative geometry inspired model can predict an effective gravitational constant as well. The plot presented in Fig. 4 exhibits the numerical results of the function $G_{\mathrm{eff}} / G$ versus the radius, $r / \sqrt{\theta}$. 


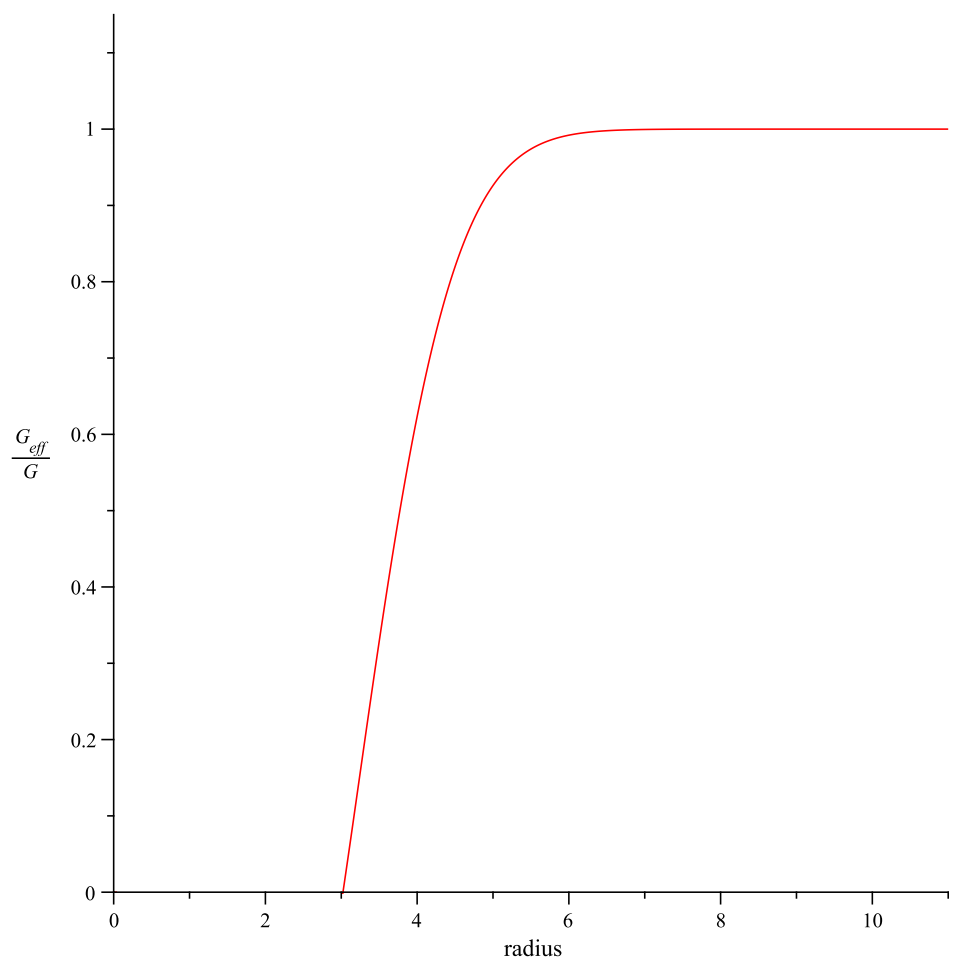

Figure 4: The function $G_{\text {eff }} / G$ versus the radius, $r / \sqrt{\theta}$. The fraction of gravitational constant deviates strongly from the unity at small scales.

As this figure shows, the lowering of the $G_{\text {eff }}$ with respect to the $G$ near the origin is clear. The appearance of a lower finite cut-off at short-scale gravity imposes a bound on any measurements to find a particle position in noncommutative geometry.

\section{Summary}

In summary, we have applied the noncommutative geometry inspired model to include the microscopic structure of spacetime in the entropic view of gravity by reason of significant coupling between the issue of entropy with the quantum spacetime structures. The entropic force in the presence of NC SdS BHs by considering the effect of smearing of the particle mass as a Gaussian distribution is derived. In this approach, the force and energy graph cut off abruptly at some finite screen radii on both small and large scales. This allows us to impose bounds on the scale of NC SdS BHs. We have shown that when one combines entropic gravity with noncommutative geometry there can be a violation of the EP for both small and large scales which signals a failure of current physical ideas. In the end, an effective gravitational constant induced by noncommutativity parameter is derived by applying noncommutative effects in Verlinde's formalism of gravity. 


\section{References}

[1] S. W. Hawking, Comm. Math. Phys. 43, 199 (1975).

[2] J. M. Bardeen, B. Carter and S. W. Hawking, Commun. Math. Phys. 31, 161 (1973).

[3] T. Jacobson, Phys. Rev. Lett. 75, 1260 (1995) arXiv:gr-qc/9504004.

[4] T. Padmanabhan, Mod. Phys. Lett. A 25, 1129 (2010) arXiv:0912.3165.

[5] E. P. Verlinde, JHEP 1104, 029 (2011) [arXiv:1001.0785].

[6] There is a large literature on this subject; see for instance, R. G. Cai, L. M. Cao and N. Ohta, Phys. Rev. D 81, 061501 (2010) arXiv:1001.3470]; M. Li and Y. Wang, Phys. Lett. B 687, 243 (2010) arXiv:1001.4466]; D. A. Easson, P. H. Frampton and G. F. Smoot, Phys. Lett. B 696, 273 (2011) arXiv:1002.4278; C. Gao, Phys. Rev. D 81, 087306 (2010) arXiv:1001.4585]; J. Kowalski-Glikman, Phys. Rev. D 81, 084038 (2010) arXiv:1002.1035]; R. G. Cai, L. M. Cao and N. Ohta, Phys. Rev. D 81, 084012 (2010) [arXiv:1002.1136]; Y. Tian and X. Wu, Phys. Rev. D 81, 104013 (2010) arXiv:1002.1275]; Y. S. Myung and Y. W. Kim, Phys. Rev. D 81, 105012 (2010) [arXiv:1002.2292]; R. A. Konoplya, Eur. Phys. J. C 69, 555 (2010) arXiv:1002.2818; ;. G. Paeng and M. Rho, Mod. Phys. Lett. A 25, 399 (2010) arXiv:1002.3022; R. Banerjee and B. R. Majhi, Phys. Rev. D 81, 124006 (2010) arXiv:1003.2312]; Y. S. Myung, Eur. Phys. J. C 71, 1549 (2011) arXiv:1003.5037; B. Koch, AIP Conf. Proc. 1232, 313 (2010) arXiv:1004.2879]; J. R. Mureika and R. B. Mann, Mod. Phys. Lett. A 26, 171 (2011) arXiv:1005.2214]; P. Nicolini, Phys. Rev. D 82, 044030 (2010) [arXiv:1005.2996]; F. R. Klinkhamer, Class. Quant. Grav. 28, 125003 (2011) arXiv:1006.2094]; R. Percacci and G. P. Vacca, Class. Quant. Grav. 27, 245026 (2010) arXiv:1008.3621]; V. V. Kiselev and S. A. Timofeev, Mod. Phys. Lett. A 26, 109 (2011) arXiv:1009.1301]; A. Kobakhidze, Phys. Rev. D 83, 021502 (2011) arXiv:1009.5414]; H. Sahlmann, Class. Quant. Grav. 28, 015006 (2011) arXiv:1010.2650]; Y. F. Cai and E. N. Saridakis, Phys. Lett. B 697 280 (2011) arXiv:1011.1245]; S. H. Hendi and A. Sheykhi, Phys. Rev. D 83, 084012 (2011) arXiv:1012.0381]; J. A. Neto, arXiv:1101.2927]; I. Sakalli, Int. J. Theor. Phys. 50, 2426 (2011) arXiv:1103.1728]; K. Nozari and S. Akhshabi, Phys. Lett. B 700, 91 (2011) arXiv:1104.4849]; T. Qiu and E. N. Saridakis, arXiv:1107.1013; 
M. Visser, JHEP 1110, 140 (2011) arXiv:1108.5240]; K. Nozari, P. Pedram and M. Molkara, arXiv:1111.2204].

[7] S. H. Mehdipour, Eur. Phys. J. Plus 127, 80 (2012) arXiv:1111.2468.

[8] S. H. Mehdipour and A. Keshavarz, Europhys. Lett. 98, 10002 (2012) arXiv:1207.0841].

[9] P. Nicolini, Int. J. Mod. Phys. A 24, 1229 (2009) arXiv:0807.1939].

[10] A. Smailagic and E. Spallucci, J. Phys. A 36, L467 (2003) arXiv:hep-th/0307217]; J. Phys. A 36, L517 (2003) arXiv:hep-th/0308193; J. Phys. A 37, 7169 (2004) arXiv:hep-th/0406174; E. Spallucci, A. Smailagic and P. Nicolini, Phys. Rev. D 73, 084004 (2006) arXiv:hep-th/0604094; R. Banerjee, B. Chakraborty, S. Ghosh, P. Mukherjee and S. Samanta, Found. Phys. 39, 1297 (2009) arXiv:0909.1000; R. Banerjee, S. Gangopadhyay and S. K. Modak, Phys. Lett. B 686, 181 (2010) arXiv:0911.2123]; L. Modesto and P. Nicolini, Phys. Rev. D 81, 104040 (2010) arXiv:0912.0220]; P. Nicolini and M. Rinaldi, Phys. Lett. B 695, 303 (2011) arXiv:0910.2860].

[11] P. Nicolini, A. Smailagic and E. Spallucci, Phys. Lett. B 632, 547 (2006) arXiv:gr-qc/0510112]; T. G. Rizzo, JHEP 0609, 021 (2006) arXiv:hep-ph/0606051; S. Ansoldi, P. Nicolini, A. Smailagic and E. Spallucci, Phys. Lett. B 645, 261 (2007) arXiv:gr-qc/0612035]; E. Spallucci, A. Smailagic and P. Nicolini, Phys. Lett. B 670, 449 (2009) arXiv:0801.3519]; Phys. Lett. B 670,449 (2009) arXiv:0801.3519]; P. Nicolini and E. Spallucci, Class. Quant. Grav. 27, 015010 (2010) arXiv:0902.4654; K. Nozari and S. H. Mehdipour, Commun. Theor. Phys. 53, 503 (2010) arXiv:0707.1080]; L. Modesto and P. Nicolini, Phys. Rev. D 82, 104035 (2010) arXiv:1005.5605.

[12] R. B. Mann and P. Nicolini, Phys. Rev. D 84, 064014 (2011) [arXiv:1102.5096].

[13] A. H. Guth, Phys. Rev. D 23, 347 (1981); A. D. Linde, Phys. Lett. B 108, 389 (1982).

[14] S. Perlmutter et al., Nature 391, 51 (1998); Astrophys. J. 517, 565 (1999) arXiv:astro-ph/9812133]; A. G. Reiss et al., Astron. J. 116, 1009 (1998) arXiv:astro-ph/9805201. 
[15] E. Chang-Young, M. Eune, K. Kimm and D. Lee, Mod. Phys. Lett. A 26, 1975 (2011) arXiv:1011.3960.

[16] R. Bousso and S. W. Hawking, Phys. Rev. D 54, 6312 (1996) arXiv:gr-qc/9606052.

[17] R. J. Adler, P. Chen and D. I. Santiago, Gen. Rel. Grav. 33, 2101 (2001) arXiv:gr-qc/0106080. 